Correspondence

Leonilde M. Moreira

Imoreira@ist.utl.pt
Received 3 May 2011

Revised 8 July 2011

Accepted 4 August 2011

\section{Mucoid morphotype variation of Burkholderia multivorans during chronic cystic fibrosis lung infection is correlated with changes in metabolism, motility, biofilm formation and virulence}

\author{
Inês N. Silva, ${ }^{1}$ Ana S. Ferreira, ${ }^{1}$ Jörg D. Becker, ${ }^{2}$ James E. A. Zlosnik, ${ }^{3}$ \\ David P. Speert, ${ }^{3}$ Ji He, ${ }^{4}$ Dalila Mil-Homens ${ }^{1}$ and Leonilde M. Moreira, ${ }^{1,5}$ \\ ${ }^{1}$ IBB - Institute for Biotechnology and Bioengineering, Centre for Biological and Chemical \\ Engineering, IST, Lisbon, Portugal \\ ${ }^{2}$ Instituto Gulbenkian de Ciência, Rua da Quinta Grande 6, 2780-156 Oeiras, Portugal \\ ${ }^{3}$ Division of Infectious and Immunological Diseases, Department of Paediatrics and Centre for \\ Understanding and Preventing Infection in Children, Faculty of Medicine, University of British \\ Columbia, 950 W 28th Ave, Vancouver, BC V5Z 4H4, Canada \\ ${ }^{4}$ Plant Biology Division, Samuel Roberts Noble Foundation, 2510 Sam Noble Parkway, Ardmore, \\ OK 73401, USA \\ ${ }^{5}$ Department of Bioengineering, Instituto Superior Técnico (IST), Av. Rovisco Pais, \\ 1049-001 Lisbon, Portugal
}

Burkholderia cepacia complex (Bcc) bacteria are opportunistic pathogens infecting hosts such as cystic fibrosis (CF) patients. Long-term Bcc infection of CF patients' airways has been associated with emergence of phenotypic variation. Here we studied two Burkholderia multivorans clonal isolates displaying different morphotypes from a chronically infected CF patient to evaluate trait development during lung infection. Expression profiling of mucoid D2095 and non-mucoid D2214 isolates revealed decreased expression of genes encoding products related to virulence-associated traits and metabolism in D2214. Furthermore, D2214 showed no exopolysaccharide production, lower motility and chemotaxis, and more biofilm formation, particularly under microaerophilic conditions, than the clonal mucoid isolate D2095. When Galleria mellonella was used as acute infection model, D2214 at a cell number of approximately $7 \times 10^{6}$ c.f.u. caused a higher survival rate than D2095, although 6 days post-infection most of the larvae were dead. Infection with the same number of cells by mucoid D2095 caused larval death by day 4 . The decreased expression of genes involved in carbon and nitrogen metabolism may reflect lower metabolic needs of D2214 caused by lack of exopolysaccharide, but also by the attenuation of pathways not required for survival. As a result, D2214 showed higher survival than D2095 in minimal medium for 28 days under aerobic conditions. Overall, adaptation during $B c c$ chronic lung infections gave rise to genotypic and phenotypic variation among isolates, contributing to their fitness while maintaining their capacity for survival in this opportunistic human niche.
Abbreviations: Bcc, Burkholderia cepacia complex; CF, cystic fibrosis; $\mathrm{CHP}$, cumene hydroperoxide; COGs, clusters of orthologous groups; DOC, sodium deoxycholate; EPS, exopolysaccharide; qRT-PCR, quantitative RT-PCR.

The Gene Expression Omnibus (GEO) accession numbers for the microarray data associated with this paper are GSE28306 (for expression data) and GSE30402 (for genomic DNA hybridization).

Two supplementary figures and four supplementary tables are available with the online version of this paper.

\section{INTRODUCTION}

Burkholderia cepacia complex (Bcc) comprises Gramnegative bacteria found in water, soil and associated with plants (Baldwin et al., 2007). They have large genomes (between 7 and $9 \mathrm{Mbp}$ ) and a number of genes ranging from 5500 to 7900 . Due to this high gene content, Bcc strains have numerous metabolic enzymes, transporters, regulatory genes and putative virulence determinants, providing them with a great competitive capacity to move 
between different niches (Holden et al., 2009). Bcc bacteria have attracted considerable interest due to infections caused in the airways of cystic fibrosis (CF) patients (Govan \& Nelson, 1992) and the difficulty of being eradicated from lung infections due to their intrinsic antibiotic resistance (Nzula et al., 2002). The outcome of $\mathrm{CF}$ lung infection by $\mathrm{Bcc}$ ranges from mild asymptomatic carriage to a rapid and fatal decline in lung function (Isles et al., 1984).

Many studies have been undertaken to understand Bcc pathogenicity, and several putative virulence factors have been identified (Leitão et al., 2010; Mahenthiralingam et al., 2005). One such factor is the exopolysaccharide (EPS) cepacian produced by a majority of Bcc strains (Zlosnik et al., 2008). In vitro studies have shown the ability of EPS to inhibit neutrophil chemotaxis and to scavange reactive oxygen species (Bylund et al., 2006). Furthermore, the EPSs produced by a Burkholderia cenocepacia clinical isolate interfered with phagocytosis of bacteria by human neutrophils and facilitated persistent bacterial infection in the BALB/c mouse model of infection (Conway et al., 2004). In the gp91 ${ }^{\text {phox-l- }}$ mouse model of infection, EPS-producing strains of Burkholderia cepacia were more virulent than non-mucoid isogenic mutants (Sousa et al., 2007). This suggests that EPS enhances bacterial virulence, but does not rule out the possibility that non-EPS-producers can cause severe infections. That is the case for B. cenocepacia and Burkholderia multivorans isolates, which, despite their lack of EPS biosynthesis ability, are highly infectious (Govan et al., 1993; Zlosnik et al., 2011). A hypothesis derived from these observations is that mucoid strains would be favoured in chronic lung infections while non-mucoid strains would be more prone to virulence in acute infections (Zlosnik et al., 2011).

Despite the evidence gained from infection models showing the importance of EPS in Bcc virulence, there is no experimental demonstration of $B c c$ bacteria producing it within the lung environment. By contrast, in Pseudomonas aeruginosa, the major pathogen of the CF lung, the initial colonization is made by environmental strains that during the course of infection develop the mucoid phenotype caused by production of alginate (Pedersen et al., 1992). This mucoid phenotypic conversion has also been reported for $B c c$ isolates recovered from CF patients chronically infected but, opposite to $P$. aeruginosa, most transitions were from mucoid to non-mucoid morphotypes (Zlosnik et al., 2008). Characterization of two B. cenocepacia sequential isolates displaying different morphotypes (mucoid vs non-mucoid) isolated from a CF patient showed differential expression of other phenotypic traits besides EPS (Conway et al., 2004; Zlosnik \& Speert, 2010). In contrast to P. aeruginosa CF lung colonization, where genetic adaptations leading to phenotypic variation are characterized (Smith et al., 2006), the diversity generated by Burkholderia persistence in the lungs is mostly unknown. It is therefore of interest to understand trait developments during Bcc persistence in CF lungs and to evaluate whether there is a typical phenotypic profile for mucoid and non-mucoid isolates during chronic infections. With that aim, the approach followed in this study was to combine transcriptional profiling using custom Burkholderia Affymetrix DNA arrays with phenotypic characterization to compare a pair of clonal B. multivorans sequential isolates recovered from a chronically infected CF patient where a mucoid to non-mucoid morphotypic transition had occurred.

\section{METHODS}

Bacterial strains and growth conditions. The Burkholderia isolates used were D2095 and D2214 (Table 1). These isolates are from a patient attending a Vancouver CF clinic as previously described (Zlosnik et al., 2008, 2011). Isolates were grown in Lennox broth (LB) or in EPS-producing medium (SM) at $37{ }^{\circ} \mathrm{C}$ (Ferreira et al., 2010). Growth under microaerophilic conditions was carried out using microaerophilic generators (GENbox microaer, bioMérieux) contained within a sealed 21 jar. For expression profiling, overnight cultures of the isolates were grown in SM medium and were diluted to an initial $\mathrm{OD}_{640}$ of 0.1 into the same medium. Triplicate $250 \mathrm{ml}$ Erlenmeyer flasks containing $100 \mathrm{ml}$ medium were cultured at $37{ }^{\circ} \mathrm{C}$ with 250 r.p.m. agitation for $17 \mathrm{~h}$.

Survival to long-term nutrient deprivation. Liquid cultures of $B$. multivorans D2095 and D2214 grown overnight in LB at $37{ }^{\circ} \mathrm{C}$, were harvested, washed with saline solution and added to $100 \mathrm{ml} \mathrm{M63}$ minimal medium without a carbon source (Sambrook \& Russell, 2001), to a final $\mathrm{OD}_{640}$ of 1.0. Cultures were incubated under aerobic conditions for 28 days at $37{ }^{\circ} \mathrm{C}$ with agitation. The number of surviving bacteria was assessed by quantification of c.f.u. on LB plates after $48 \mathrm{~h}$ incubation at $37^{\circ} \mathrm{C}$.

Phenotypic assays. EPS production was assessed based on the ethanol-precipitated polysaccharide dry weight recovered from $100 \mathrm{ml}$ bacterial cultures grown in liquid SM over 3 days at $37^{\circ} \mathrm{C}$, as described previously (Ferreira et al., 2007). Antimicrobial

Table 1. Mucoid phenotype displayed by B. multivorans sequential isolates recovered from a chronically infected CF patient

\begin{tabular}{|lllc|}
\hline Isolate & Date of isolation & Source & EPS score \\
\hline C5568 & 30 November 1993 & Sputum & +++ \\
C6558 & 26 May 1995 & Throat swab & ++ \\
C7148 & 14 June 1996 & Sputum & +++ \\
C7149 & 14 June 1996 & Sputum & ++ \\
C7637 & 06 June 1997 & Sputum & + \\
C8179 & 20 June 1998 & Sputum & ++ \\
C9326 & 23 September 2000 & Sputum & ++ \\
D0089 & 29 March 2002 & Throat swab & ++ \\
D1782 & 03 October 2005 & Throat swab & +++ \\
D2094 & 01 June 2006 & Throat swab & +++ \\
D2095 & 01 June 2006 & Throat swab & +++ \\
D2214 & 09 November 2006 & Sputum & - \\
& & & \\
\hline
\end{tabular}

${ }^{*}$ Classification according to Zlosnik et al. (2008): very mucoid isolates are scored as ++ or +++ ; slightly mucoid are scored as + ; nonmucoid isolates are scored as -. 
susceptibility tests were based on the agar disc diffusion method (Bauer et al., 1966) against piperacillin $(100 \mu \mathrm{g})$, piperacillin $(75 \mu \mathrm{g})$ plus tazobactam $(10 \mu \mathrm{g})$, ciprofloxacin $(5 \mu \mathrm{g})$, ceftazidime $(30 \mu \mathrm{g})$ and amikacin $(30 \mu \mathrm{g})$. The discs were applied onto the surface of Mueller-Hinton (Difco Laboratories) agar plates previously inoculated with $100 \mu \mathrm{l}$ of bacterial cultures grown overnight in SM, at $37{ }^{\circ} \mathrm{C}$ and diluted to $\mathrm{OD}_{640} 0.1$. Growth inhibition diameter was measured after $24 \mathrm{~h}$ incubation at $37^{\circ} \mathrm{C}$. For zone inhibition assays, bacteria were grown in SM medium and $100 \mu$ of a culture of $\mathrm{OD}_{640}$ 1.0 were spread onto SM plates. Sterile paper discs $6 \mathrm{~mm}$ in diameter were placed on the agar surface. A total of $20 \mu \mathrm{l}$ SDS $(10 \% \mathrm{w} / \mathrm{v})$, sodium deoxycholate (DOC) $(5 \% \mathrm{w} / \mathrm{v})$, cumene hydroperoxide (CHP) $(10 \% \mathrm{v} / \mathrm{v})$ and $\mathrm{H}_{2} \mathrm{O}_{2}(30 \% \mathrm{v} / \mathrm{v})$ was pipetted onto separate discs. The plates were incubated for $24 \mathrm{~h}$ at $37^{\circ} \mathrm{C}$ and the zone of growth inhibition measured. Biofilm formation assays were performed as previously described (Ferreira et al., 2007). Bacteria were grown in SM medium at $37{ }^{\circ} \mathrm{C}$ to mid-exponential phase, diluted to $\mathrm{OD}_{640} 0.05$, and $200 \mu \mathrm{l}$ of these cell suspensions were inoculated into wells of a 96-well polystyrene microtitre plate. Plates were incubated at $37{ }^{\circ} \mathrm{C}$ without agitation under either normal atmosphere or microaerophilic conditions inside anaerobic jars. The biofilm was stained with crystal violet solution, followed by dye solubilization with ethanol and measurement of the solution's $A_{590}$ using a microplate reader as previously described (Ferreira et al., 2007). Swimming plates with $0.3 \%(\mathrm{w} / \mathrm{v})$ Bacto agar (Difco) and swarming plates with $0.5 \%(\mathrm{w} / \mathrm{v})$ Noble agar (Difco) were prepared using Broomfield medium [0.04\% (w/v) tryptone; $0.01 \%(\mathrm{w} / \mathrm{v})$ yeast extract; and $\left.0.0067 \%(\mathrm{w} / \mathrm{v}) \mathrm{CaCl}_{2}\right]$ and $\mathrm{LB}$ with $0.5 \%(\mathrm{w} / \mathrm{v})$ glucose, respectively. For estimation of motility, overnight SM bacterial cultures $(5 \mu \mathrm{l})$ were inoculated onto the agar surface and incubated at $37^{\circ} \mathrm{C}$ for $48 \mathrm{~h}$ followed by measurement of colony diameter.

Virulence determination in Galleria mellonella. Killing assays were performed as described by Seed \& Dennis (2008). Larvae were given injections of an increasing number of bacteria ranging from $7 \times 10^{3}$ to $7 \times 10^{6}$ c.f.u. diluted in $10 \mathrm{mM} \mathrm{MgSO}_{4}$ with $1.2 \mathrm{mg}$ ampicillin $\mathrm{ml}^{-1}$ and the survival rate was evaluated for 6 days postinfection. As a negative control, $10 \mathrm{mM} \mathrm{MgSO}$ with $1.2 \mathrm{mg}$ ampicillin $\mathrm{ml}^{-1}$ was used. Triplicates of 10 larvae were used in each experiment.

Bacterial genotyping. Sample preparation and PFGE were performed as previously described (Moreira et al., 1997). Prior to PFGE, immobilized DNA was digested with $20 \mathrm{U}$ SpeI restriction endonuclease before being loaded onto a $1 \%(\mathrm{w} / \mathrm{v})$ agarose gel in $0.5 \times$ Tris/borate/EDTA buffer. PFGE was carried out with a Gene Navigator apparatus (Pharmacia-LKB) at $180 \mathrm{~V}$, using 5-120 s pulse times for $22 \mathrm{~h}$.

recA gene amplification and RFLP analysis was performed to confirm that the two isolates belonged to B. multivorans. The primers used for recA amplification were BCR1 (TGACCGCCGAGAAGAGCAA) and BCR2 (CTCTTCTTCGTCCATCGCCTC), followed by HaeIII digestion of the $1043 \mathrm{bp}$ amplification product.

Custom Burkholderia microarray design. The nucleotide sequences used for microarray design were from B. multivorans ATCC 17616 and B. cenocepacia J2315 genomes present in GenBank. Sequences corresponding to coding regions from the two genomes were aligned against each other based on BLASTN (Altschul et al., 1997). Using the web tools available through the Personal BLAST Navigator (PLAN) (He et al., 2007) system, genes with $>90 \%$ identity at the nucleotide level were targeted and exported in tabular format for common probe selection. The parameters chosen for the custom array design were as follows: $11 \mu \mathrm{m}$ feature size, standard $12.8 \mathrm{~mm}$ array format, prokaryotic antisense target type, perfectmatch probes only, full-length sequence for probe selection, and eight as the minimum number of acceptable probes per sequence. The types of probe sets represented are: 10032 unique; 3342 gene (_a); 247 identical (_s); and 1203 mixed (_x). B. cenocepacia J2315 genes are represented by 4790 unique probes; 3210 gene (_a); 136 identical (_s); and 676 mixed (_x). B. multivorans ATCC 17616 genes are represented by 5099 unique probes; 3268 gene (_a); 158 identical (_s); and 766 mixed (_x). A total of 280 probe sets representing 213 regions of the B. cenocepacia $\mathrm{J} 2315$ genome encoding putative noncoding small RNAs as predicted elsewhere (Coenye et al., 2007) were also included. Probe gene coverage is 99.0 and $99.8 \%$ for $B$. cenocepacia J2315 and B. multivorans ATCC 17616, respectively.

Isolation and processing of RNA and DNA samples. For RNA analysis bacterial cells were resuspended in RNAprotect bacteria reagent (Qiagen), and total RNA extraction was carried out using the RNeasy MiniKit (Qiagen) by following the manufacturer's recommendations. RNA integrity was checked on an Agilent 2100 Bioanalyser using an RNA Nano assay. RNA was processed for use on Affymetrix custom dual-species Burkholderia arrays, according to the manufacturer's Prokaryotic Target Preparation Assay. Briefly, $10 \mu \mathrm{g}$ total RNA containing spiked-in poly(A) RNA controls (GeneChip Poly-A RNA Control kit; Affymetrix) was used in a reverse transcription reaction with random primers (Invitrogen Life Technologies) to generate first-strand cDNA. After removal of RNA, $2 \mu \mathrm{g}$ cDNA was fragmented with DNase and end-labelled with biotin using terminal polynucleotidyltransferase (GeneChip WT Terminal Labelling kit, Affymetrix). Size distribution of the fragmented and end-labelled cDNA was assessed using an Agilent 2100 Bioanalyser. Samples $(2 \mu \mathrm{g})$ of end-labelled fragmented cDNA were used in a $200 \mu$ hybridization cocktail containing added hybridization controls and hybridized on arrays for $16 \mathrm{~h}$ at $50{ }^{\circ} \mathrm{C}$. Modified posthybridization wash and double-stain protocols (FLEX450_0005; GeneChip HWS kit, Affymetrix) were used on an Affymetrix GeneChip Fluidics Station 450. Arrays were scanned on an Affymetrix GeneChip scanner 3000 7G. Biological triplicates of RNA from each bacterial culture were processed and analysed.

For DNA analysis bacterial cells were grown in liquid LB for $15 \mathrm{~h}$, followed by DNA extraction using the DNeasy blood and tissue kit (Qiagen). A total of $1.5 \mu \mathrm{g}$ genomic DNA per sample was labelled using the Bioprime DNA labelling System (Invitrogen) following a strategy for genomic DNA hybridizations to GeneChips developed by Hammond et al., (2005). Cleanup was performed using MinElute PCR Purification kit (Qiagen) and quality was checked on an Agilent 2100 Bioanalyser using a DNA 1000 assay. Five micrograms of each sample was analysed on Affymetrix custom dual-species Burkholderia arrays following the protocol described above for RNA samples. Duplicates of DNA from each bacterial culture were processed and analysed.

Microarray analysis. For RNA analysis scanned arrays were analysed with Affymetrix Expression Console software to assure that all quality parameters were in the recommended range. Subsequent analysis was carried out with DNA-Chip Analyser 2008. First a digital mask was applied, leaving for analysis only the 9291 probe sets representing $B$. multivorans ATCC 17616 transcripts. Then the six arrays were normalized to a baseline array with median CEL intensity by applying an Invariant Set Normalization Method (Li \& Wong, 2001a). Normalized CEL intensities of the arrays were used to obtain model-based gene expression indices based on a Perfect Match (PM)only model (Li \& Wong, 2001b). Replicate data (triplicates) for each bacterial isolate were weighted gene-wise by using inverse squared standard error as weights. All genes compared were considered to be differentially expressed if the $90 \%$ lower confidence bound of the fold change (LCB) between experiment and baseline was above 1.2, resulting in 392 differentially expressed transcripts with a median false discovery rate of $4.7 \%$. 
For DNA a separate analysis was performed for the four arrays following the steps described above for RNA. An LCB cut-off of 1.5 in combination with a consistent hybridization signal above 10 in the two replicas of at least one strain was chosen, resulting in 52 genomic loci showing significantly different hybridization signals.

Quantitative real-time RT-PCR (qRT-PCR). DNA microarray data were validated by qRT-PCR as previously described (Ferreira et al., 2010). Total RNA was used in a reverse transcription reaction with TaqMan Reverse Transcription Reagents (Applied Biosystems). qRTPCR amplification of each gene (for primer sequences see Supplementary Table S1, available with the online version of this paper) was performed with a model 7500 thermocycler (Applied Biosystems). The expression ratio of the target genes relative to the reference gene $\operatorname{trp} B$, which showed no variation in the transcription abundance under the conditions tested, was determined. Relative quantification of gene expression by real-time qRT-PCR was determined using the $\Delta \Delta C_{\mathrm{t}}$ method (Pfaffl, 2001).

\section{RESULTS}

\section{Mucoid phenotype assessment in sequential isolates of $B$. multivorans}

During a 13-year period 12 isolates were recovered from a CF patient persistently infected with Burkholderia (Table 1). This patient, who is still alive, had simultaneously yielded cultures of other bacteria such as Staphylococcus aureus and Pseudomonas aeruginosa, but at the times when the last six isolates were obtained only $B$. multivorans was present. These isolates were analysed by randomly amplified polymorphic DNA (RAPD) and all of them belonged to RAPD group BM019 , corresponding to the same $B$. multivorans strain (Zlosnik et al., 2008). The assessment of EPS production in yeast extract mannitol (YEM) agar showed that isolates recovered from November 1993 until June 2006 were mucoid, although some variation in the amount of EPS was observed (Table 1). Interestingly, the last isolate (D2214) was completely non-mucoid and represents a mucoid to non-mucoid transition. Additional confirmation that mucoid D2095 and non-mucoid D2214 are indeed clonal came from PFGE of genomic DNA digested with SpeI, since the DNA fragment pattern obtained was almost identical (see Supplementary Fig. S1). Specific primers to amplify the recA gene from Burkholderia followed by restriction analysis with HaeIII also confirmed that both isolates are indeed from the species B. multivorans (results not shown).

Isolates D2095 and D2214 were chosen here to gain insights into phenotypic variation during $B c c$ chronic lung infection and in particular to assess differences between the mucoid and non-mucoid morphotypes. Two of the analysed features were growth behaviour under aerobic conditions and production of EPS in SM liquid medium. The two isolates displayed identical growth rates (Fig. 1a), but assessment of EPS biosynthesis confirmed that D2095 produces approximately $10 \mathrm{~g} \mathrm{EPS} \mathrm{l}^{-1}$ after $72 \mathrm{~h}$ of growth, while no EPS was recovered from the supernatant of D2214 (Fig. 1b), confirming the morphotypes obtained previously in YEM (Zlosnik et al., 2008).
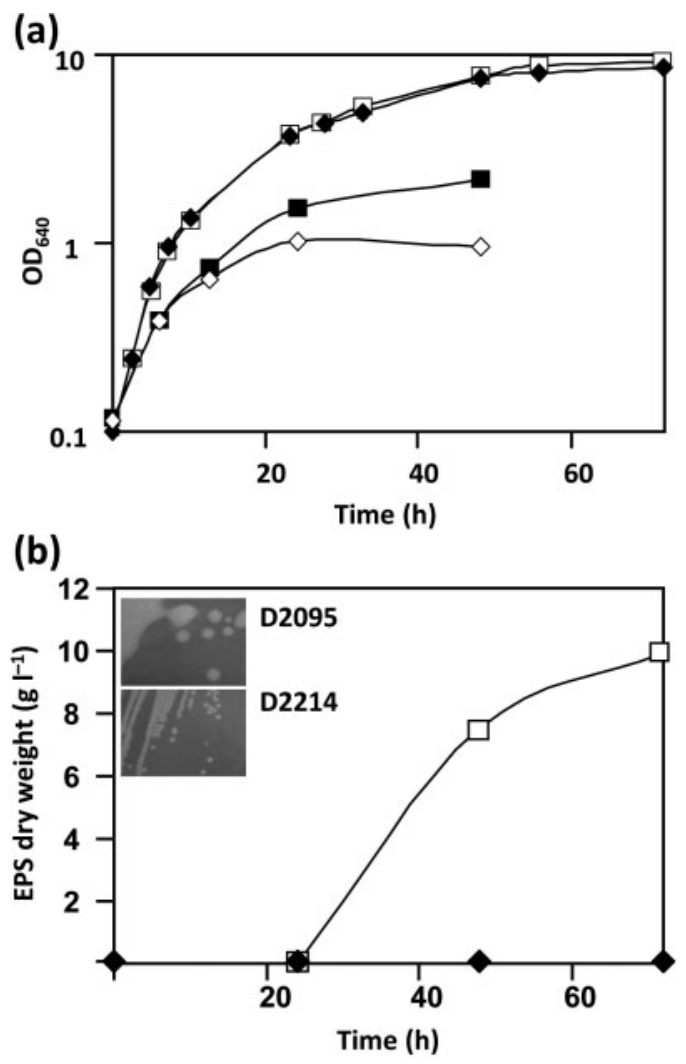

Fig. 1. Growth curves (a) and EPS production (b) for $B$. multivorans D2095 under aerobic ( $\square$ ) or microaerophilic ( $\boldsymbol{\square}$ ) conditions and D2214 under aerobic $(\diamond)$ or microaerophilic $(\diamond)$ conditions, grown in $\mathrm{SM}$ medium at $37^{\circ} \mathrm{C}$. The standard deviation for both panels was below $5 \%$ of the mean. The data are based on mean values from the results of at least three independent cell cultivations.

\section{Expression profiling among sequential mucoid and non-mucoid isolates of $B$. multivorans}

To understand the differences between isolates D2095 and D2214, transcriptional profiling was performed and data mapped to cellular processes and metabolic pathways. For that purpose, a dual-species custom Burkholderia microarray (Bcc1sa520656F) was designed and produced by Affymetrix as described in Methods. One of the species represented in the array is B. multivorans ATCC 17616 and its coding regions are represented by 5099 unique probes, 3268 gene (_a), 158 identical (_s) and 766 mixed (_x), with gene coverage of $99.8 \%$. To determine which probes from the array hybridize differentially between the genomes of $B$. multivorans D2095 and D2214, both genomic DNAs were extracted, biotin-labelled and hybridized in duplicate to the microarray Bcc1sa520656F. Genomic DNA hybridization intensities from D2214 were compared to those of D2095 using dChip ( $\geqslant 1.5$-fold change lower confidence bound and hybridization signal above 10), resulting in 52 genes 
with differential hybridization (see Supplementary Table S2). The majority of these genes are located very close to each other on chromosome 2. Genes Bmul_4779 to Bmul_4788 seem to be absent from the genome of isolate D2214 as shown by the image of the probe set intensities in both isolates for gene Bmul_4779 (Supplementary Fig. S2a). These genes encode putative ABC-transporter-related proteins and a transcriptional regulator of the MarR family. Another region most likely absent from D2214 comprises genes Bmul_4804 to Bmul_4812 with the exception of Bmul_4807, putatively involved in the degradation pathway of phthalate. Finally, the largest group of genes, comprising Bmul_4834 to Bmul_4874 (with a few exceptions), seem to be duplicated in the D2214 genome. Since the probe sets corresponding to these genes showed intense hybridization signals (for an example see Supplementary Fig. S2b), it is unlikely that it corresponds to a deletion in the D2095 genome. All of this last group of genes encode proteins putatively related to bacteriophages. From this genomic hybridization experiment we demonstrate that indeed some genetic alterations took place during lung colonization by these isolates.

To carry out transcription profiling, RNA was extracted from the mucoid D2095 and the non-mucoid D2214 isolates grown in SM medium until early stationary phase, corresponding to a period where high-molecular-mass EPS is not yet recovered from the culture supernatant. The expression profile of D2214 when compared with D2095 using dChip ( $\geqslant 1.2$-fold change lower confidence bound with a resulting false discovery rate of $\leqslant 4.7 \%$ ) showed 113 genes with significantly increased expression (see Supplementary Table S3) and 279 genes with significantly decreased expression (see Supplementary Table S4). The search for common genes between the expression data and the genomic hybridization data showed 22 genes, 8 increased in both datasets and 14 decreased (Fig. 2). These genes were excluded from our expression analysis, leaving 105 genes with significantly increased expression and 265 with significantly decreased expression (Fig. 2). The differentially expressed genes were assigned to clusters of orthologous groups (COGs) as shown in Fig. 3. No particular categories with a large number of genes with significantly increased expression were obtained (Fig. 3, grey bars). Categories with a large number of genes with significantly decreased expression were cell wall/membrane/envelope biogenesis and cell motility (Fig. 3, black bars). There was no preferential distribution of differentially expressed genes within the three replicons, with $6.8,6.8$ and $3.5 \%$ of the genes from chromosome 1,2 and 3, respectively, being differentially expressed.

To confirm the data obtained by microarray analysis, expression of seven representative genes from COGs $\mathrm{K}, \mathrm{M}$, $\mathrm{N}$ and $\mathrm{T}$ was analysed by qRT-PCR. The results obtained were in good agreement with the microarray data although the fold change for most of the genes was higher when expression was analysed by qRT-PCR (Table 2).

\section{Genes involved in virulence traits}

In general, a decrease in transcripts of genes encoding virulence-associated traits, such as motility, chemotaxis, type VI secretion and antibiotic resistance, was observed in D2214 (Table 3, Supplementary Table S4). Concerning motility and chemotaxis, nearly all $\mathrm{flh}$, flg, mot, fli and che genes showed decreased expression in D2214 when compared with D2095 (Table 3). The sole exceptions were the filament-encoding genes fliCD and fliOPQR, whose gene products are involved in flagellar assembly, with no significant change. Besides the decreased expression of several genes encoding methylaccepting chemotaxis proteins, the D2214 isolate also

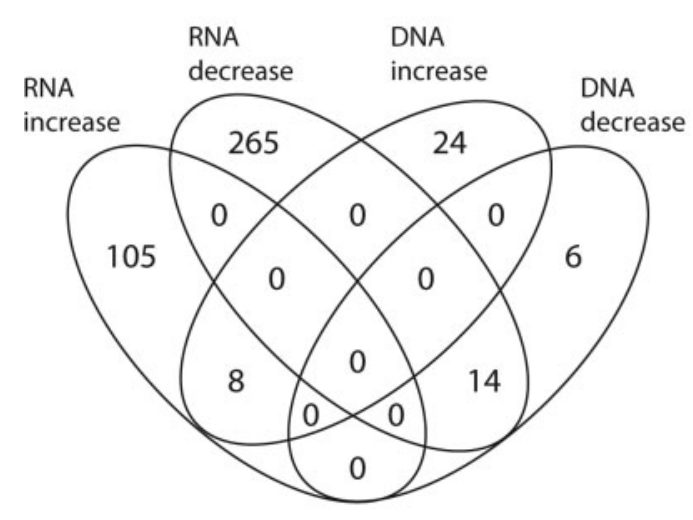

Genes removed from the expression data

$\begin{array}{ll}\text { Phage related }(\uparrow): & \text { ABC transporter }(\downarrow) \text { : } \\ \text { Bmul_4836 } & \text { Bmul_4779 } \\ \text { Bmul_4838 } & \text { Bmul_4781 } \\ \text { Bmul_4839 } & \text { Bmul_4783 } \\ \text { Bmul_4853 } & \text { Bmul_4785 } \\ \text { Bmul_4854 } & \text { Bmul_4787 } \\ \text { Bmul_4857 } & \text { Bmul_4788 } \\ \text { Bmul_4858 } & \\ \text { Bmul_4859 } & \text { Phthalate degradation }(\downarrow) \text { : } \\ & \text { Bmul_4804 } \\ & \text { Bmul_4805 } \\ & \text { Bmul_4806 } \\ & \text { Bmul_4808 } \\ & \text { Bmul_4809 } \\ & \text { Bmul_4810 } \\ & \text { Bmul_4811 } \\ & \text { Bmul_4812 }\end{array}$

Fig. 2. Diagram representing the intersection between the differentially expressed genes and the genomic DNA differential hybridization when D2214 was compared with D2095. Genes excluded from our expression dataset are indicated. 


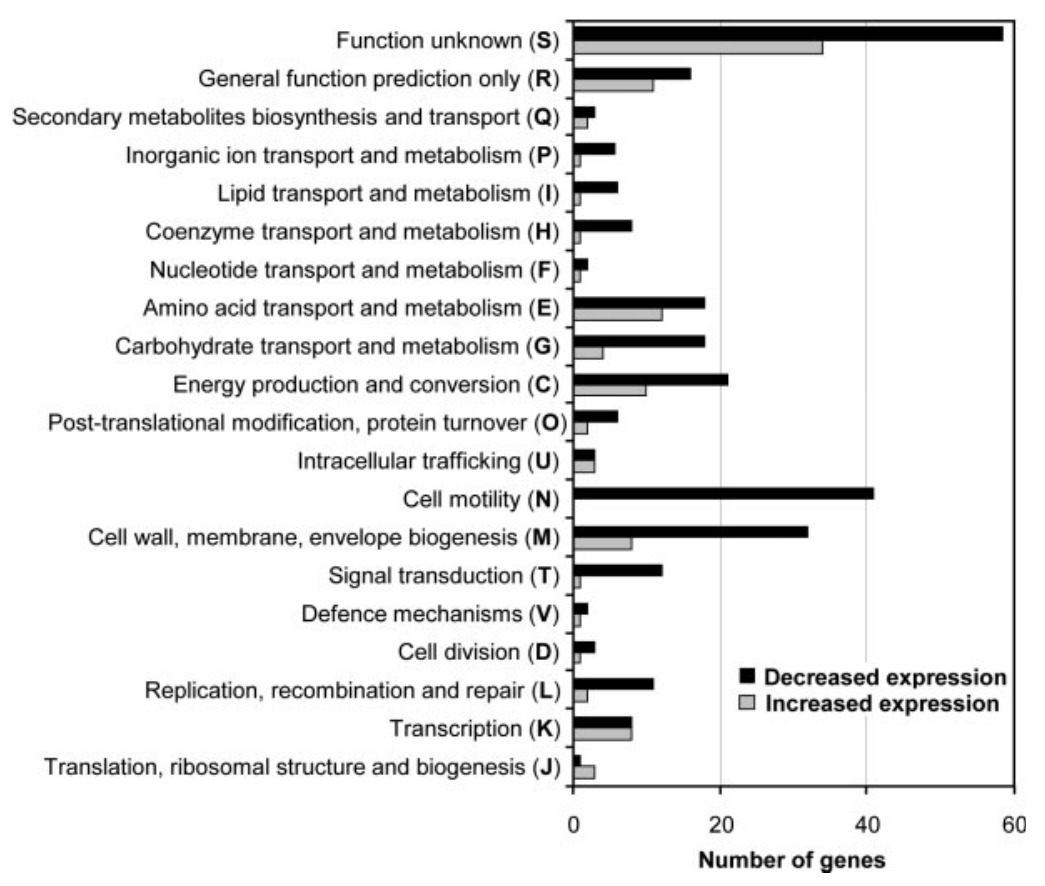

Fig. 3. Functional distribution into COGs of genes with altered expression when the nonmucoid B. multivorans D2214 isolate was compared with the mucoid isolate D2095. A total of 370 genes with statistically significantly altered expression were obtained by using a custom Affymetrix Burkholderia GeneChip. displayed decreased expression of the aer gene encoding a protein homologous to the aerotaxis (oxygen-sensing) receptor Aer from other bacteria. Through control of the flagellar motor, Aer sensing enables motile bacteria to avoid niches where carbon deficiency, hypoxia or other insults would limit energy production (Taylor, 2007). To confirm the microarray data, swimming and swarming motilities were assessed by quantitative plate assays. Under the conditions tested, D2214 showed a reduction of $15 \%$ of swimming motility and $45 \%$ of swarming motility when the size of the colonies formed was compared with those of the D2095 isolate (Fig. 4a).

Another set of genes with significantly decreased expression in isolate D2214 were those encoding products required for type VI secretion: 9 of the 18 genes annotated as being involved in type VI secretion showed differential expression (Table 3). Although genes encoding type VI secretion system components are poorly expressed in vitro, this

Table 2. Quantitative real-time RT-PCR analysis performed in B. multivorans D2095 and D2214 cells

\begin{tabular}{|lcc|}
\hline Gene & $\begin{array}{c}\text { Microarray lower } \\
\text { bound of fold change }\end{array}$ & $\begin{array}{c}\text { Real-time fold } \\
\text { change } \pm \text { SD }\end{array}$ \\
\hline Bmul_0161 (flhC) & -2.2 & $-3.5 \pm 1.6$ \\
Bmul_0195 (rlpA) & 1.8 & $1.2 \pm 0.1$ \\
Bmul_3013 (flgI) & -2.3 & $-3.3 \pm 0.2$ \\
Bmul_4264 & -1.5 & $-3.1 \pm 0.5$ \\
Bmul_4265 & -1.5 & $-3.5 \pm 1.2$ \\
Bmul_4613 (bceM) & -3.4 & $-6.2 \pm 1.3$ \\
Bmul_4914 (bceG) & -18.3 & $-8.8 \pm 0.1$ \\
\hline
\end{tabular}

type of transport was discovered as a novel factor of $B$. cenocepacia survival in a rat model of chronic lung infection (Aubert et al., 2010; Hunt et al., 2004). In a recent study, Schwarz et al. (2010) proposed a broader physiological significance of type VI secretion in providing defence against simple eukaryotic cells and other bacteria in the environment. Co-inoculation of D2095 and D2214 isolates in SM liquid medium and incubation for 3 days at $37^{\circ} \mathrm{C}$ followed by mucoid versus non-mucoid c.f.u. determination indicated that neither of the strains predominated over the other (data not shown). As controls, single cultures were grown under the same tested conditions to demonstrate that no morphotype variation occurred during that period of time.

The expression of genes Bmul_1515 and Bmul_6008, encoding two putative $\beta$-lactamases, was significantly decreased in isolate D2214 (Table 3). This was consistent with the intrinsic antibiotic resistance pattern obtained by using the antibiotic disc assay. The inhibition haloes obtained in the presence of ciprofloxacin, ceftazidime and amikacin were very similar for both isolates, but for the $\beta$ lactam piperacillin alone or with tazobactam, isolate D2095 was significantly more resistant than D2214 (Fig. 4b). The same result was obtained in liquid cultures supplemented with each of the antibiotics under test (results not shown).

Other genes encoding putative virulence factors which had decreased expression in D2214 were Bmul_3709, Bmul_3710 and Bmul_3712, directing the synthesis of a haemolysin-type calcium-binding protein and a type-I transporter from the HlyD family (Table 3 ), and the acidphosphatase-encoding gene Bmul_4180. The protein encoded by Bmul_4180 is homologous to AcpA from Burkholderia pseudomallei and, although this protein is 
Table 3. Selection of a set of differentially expressed genes when comparing the B. multivorans D2214 transcriptome with that of D2095, separated by functional groups

\begin{tabular}{|c|c|c|c|c|}
\hline Functional class & Gene identifier & LB-FC $^{*}$ & Gene name & Description \\
\hline \multirow{17}{*}{$\begin{array}{l}\text { Flagellar synthesis, motility and } \\
\text { chemotaxis } \dagger\end{array}$} & Bmul_0043 & -1.8 & fliM & Flagellar motor switch protein FliM \\
\hline & Bmul_0165 & -1.9 & cheA & CheA signal transduction histidine kinase \\
\hline & Bmul_0168 & -1.8 & cheR & MCP methyltransferase, CheR-type \\
\hline & Bmul_0169 & -2.1 & cheD & CheD \\
\hline & Bmul_0177 & -1.8 & $f l h F$ & GTP-binding signal recognition particle SRP54 G-domain \\
\hline & Bmul_0178 & -1.9 & $f l h G$ & Flagellar biosynthesis protein, FlhG \\
\hline & Bmul_3010 & -1.9 & $f l g K$ & Flagellar hook-associated protein FlgK \\
\hline & Bmul_3013 & -2.3 & flgI & Flagellar basal body P-ring protein \\
\hline & Bmul_3014 & -1.9 & $f \lg H$ & Flagellar basal body L-ring protein \\
\hline & Bmul_3015 & -1.9 & $f \lg G$ & Flagellar basal-body rod protein FlgG \\
\hline & Bmul_3018 & -1.9 & $f l g D$ & Flagellar hook capping protein \\
\hline & Bmul_3020 & -1.7 & $f l g B$ & Flagellar basal-body rod protein FlgB \\
\hline & Bmul_3023 & -2.0 & $f l g N$ & FlgN family protein \\
\hline & Bmul_3059 & -1.8 & flis & Flagellar protein FliS \\
\hline & Bmul_3061 & -1.9 & fliF & Flagellar M-ring protein FliF \\
\hline & Bmul_3062 & -1.9 & fliG & Flagellar motor switch protein FliG \\
\hline & Bmul_3362 & -1.7 & aer & Methyl-accepting chemotaxis sensory transducer \\
\hline \multirow[t]{15}{*}{ Virulence and pathogenesis } & Bmul_1515 & -2.2 & - & $\beta$-Lactamase domain protein \\
\hline & Bmul_2854 & -1.3 & paaI & Phenylacetic acid degradation protein $\mathrm{PaaD}$ \\
\hline & Bmul_2923 & -1.3 & $b c s E$ & Type VI secretion-associated protein, ImpA family \\
\hline & Bmul_2924 & -1.2 & $b c s F$ & Type VI secretion ATPase, ClpV1 family \\
\hline & Bmul_2925 & -1.4 & $b c s G$ & Type VI secretion protein, VC_A0111 family \\
\hline & Bmul_2927 & -1.6 & $b c s I$ & Type VI secretion system lysozyme-related protein \\
\hline & Bmul_2928 & -1.3 & $b c s J$ & Type VI secretion system effector, Hcp1 family \\
\hline & Bmul_2929 & -1.4 & $b c s K$ & Type VI secretion protein, EvpB/VC_A0108 family \\
\hline & Bmul_2930 & -1.2 & $b c s L$ & Type VI secretion protein \\
\hline & Bmul_2931 & -1.3 & $b c s M$ & TPR repeat-containing protein \\
\hline & Bmul_2934 & -1.2 & $b c s P$ & Hypothetical protein (type VI) \\
\hline & Bmul_3709 & -1.4 & $a d h$ & Haemolysin-type calcium-binding region \\
\hline & Bmul_3710 & -1.7 & - & Outer membrane efflux protein \\
\hline & Bmul_3712 & -1.2 & - & Type I secretion membrane fusion protein, HlyD family \\
\hline & Bmul_6008 & -1.4 & $\operatorname{ampC}$ & Beta-lactamase \\
\hline \multirow[t]{10}{*}{ Cell wall/membrane biogenesis } & Bmul_0303 & -1.4 & ompW & OmpW family protein \\
\hline & Bmul_4605 & -10.1 & bceT & UTP-glucose-1-phosphate uridylyltransferase \\
\hline & Bmul_4612 & -6.3 & $b c e N$ & GDP-mannose 4,6-dehydratase \\
\hline & Bmul_4913 & -8.2 & $b c e H$ & Glycosyltransferase-like protein \\
\hline & Bmul_4914 & -18.3 & bce $G$ & Glycosyltransferase family 2 \\
\hline & Bmul_4915 & -8.0 & $b c e F$ & Phosphotyrosine autokinase \\
\hline & Bmul_4916 & -8.4 & $b c e E$ & Polysaccharide biosynthesis protein \\
\hline & Bmul_4918 & -10.2 & bceC & Nucleotide sugar dehydrogenase \\
\hline & Bmul_4919 & -4.2 & $b c e B$ & Undecaprenyl-phosphate glucose phosphotransferase \\
\hline & Bmul_4920 & -8.1 & $b c e A$ & Mannose-1P guanylyltransferase/mannose- $6 \mathrm{P}$ isomerase \\
\hline \multirow[t]{5}{*}{ Transcription regulators } & Bmul_0179 & -1.9 & fliA & RNA polymerase, sigma 28 subunit, FliA/WhiG \\
\hline & Bmul_2557 & -2.1 & - & Transcriptional regulator, LysR family \\
\hline & Bmul_3022 & -1.7 & $f \lg M$ & Anti-sigma-28 factor, FlgM \\
\hline & Bmul_3720 & 2.9 & - & Transcriptional regulator, XRE family \\
\hline & Bmul_3782 & -1.8 & - & Transcriptional regulator, AraC family \\
\hline \multirow[t]{8}{*}{ Regulatory/signal transduction } & Bmul_0160 & -1.5 & $f l h D$ & Flagellar transcriptional activator \\
\hline & Bmul_0161 & -2.2 & $f l h C$ & Flagellar transcriptional activator FlhC \\
\hline & Bmul_0171 & -1.7 & cheY & Response regulator receiver protein \\
\hline & Bmul_2116 & -1.5 & - & Diguanylate cyclase/phosphodiesterase with PAS/PAC sensor \\
\hline & Bmul_3168 & -1.5 & - & Response regulator receiver protein \\
\hline & Bmul_4264 & -1.5 & - & Response regulator receiver protein \\
\hline & Bmul_4265 & -1.5 & fnr & Transcriptional regulator, Crp/Fnr family \\
\hline & Bmul_5122 & -1.2 & - & Diguanylate cyclase/phosphodiesterase with PAS/PAC sensor \\
\hline
\end{tabular}


Table 3. cont.

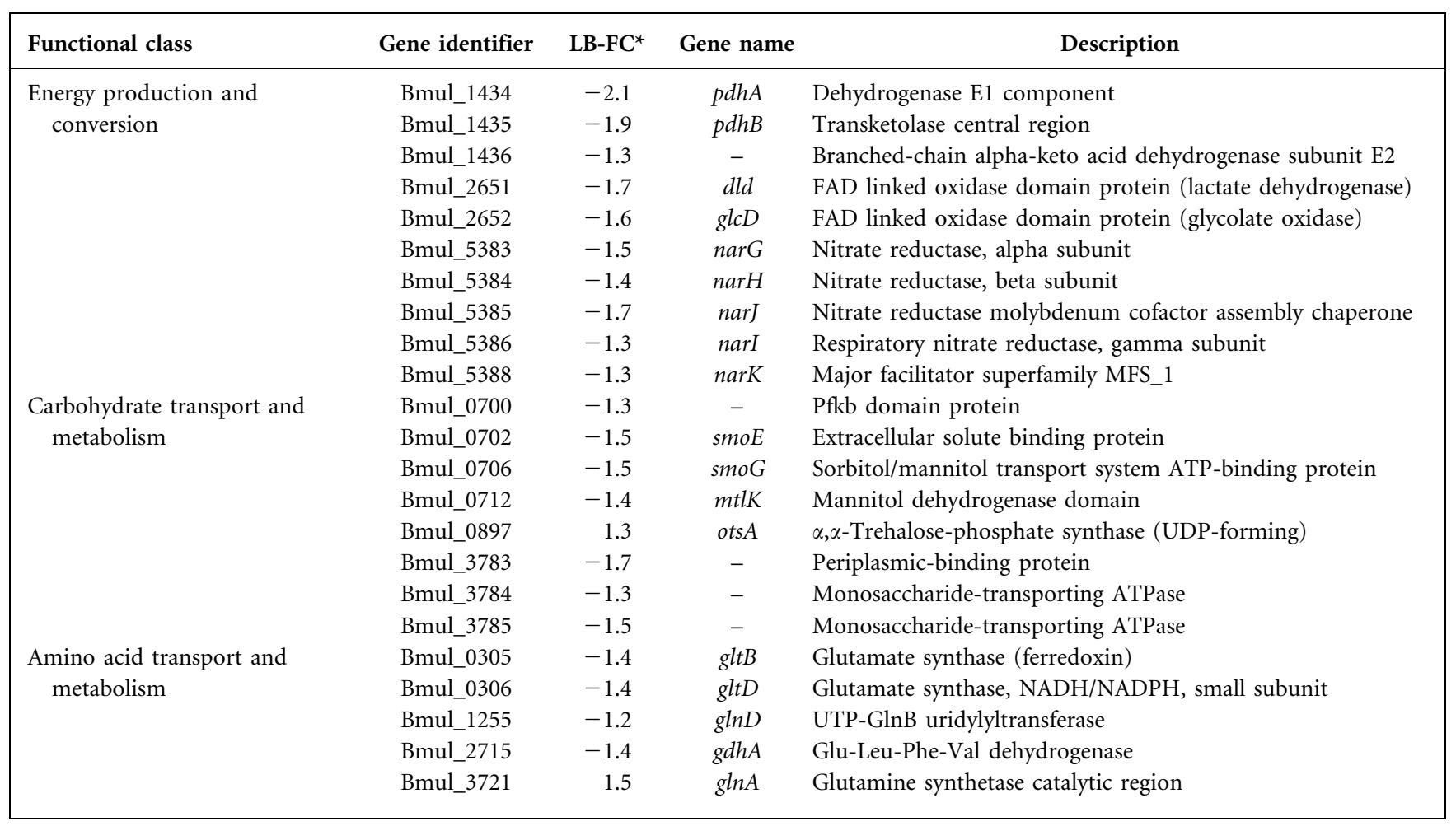

${ }^{\star}$ LB-FC, lower bound of fold-change;

$\dagger$ Only genes with a lower bound of fold-change above 1.7 are shown.

$\ddagger$ The $b c e$ genes shown are the ones with a lower bound of fold-change above 4.0.

considered a putative virulence factor, the disruption of the acp $A$ gene from $B$. pseudomallei did not significantly reduce the virulence of this organism (Burtnick et al., 2001).

$B c c$ bacteria are most probably subjected to oxidative stress in the lung, as caused by the presence of abundant neutrophils (Downey et al., 2009). Although none of the genes usually found to be involved in combating oxidative stress were differentially expressed in our dataset, the behaviour of both isolates under this type of stress was evaluated. We compared the resistance of isolates D2095 and D2214 grown in the presence of cumene hydroperoxide (CHP) and $\mathrm{H}_{2} \mathrm{O}_{2}$ (Fig. 4c); the results confirmed that the isolates react with the same magnitude to oxidative stress. Stress induced by SDS and DOC was also tested and the results showed a slight increase in susceptibility by D2214 (Fig. 4c).

Biofilm formation contributes to infection by protecting the bacteria from the host immune defence (Costerton et al., 1999). To assess biofilm formation on an abiotic surface by D2095 and D2214, a quantitative assay using crystal violet was used. After $48 \mathrm{~h}$ incubation under aerophilic conditions, the adherence level by isolate D2214 was slightly greater than that of D2095, as determined from the absorbance of the solubilized dye (Fig. 5a). Interestingly, when the biofilm was formed under microaerophilic conditions, this difference became much more prominent, suggesting that a reduction of the oxygen level favoured D2214 biofilm formation (Fig. 5a).

Galleria mellonella was used as an acute model of infection to compare the virulence of the mucoid isolate D2095 and the non-mucoid isolate D2214. Several inocula ranging from $7 \times 10^{3}$ to $7 \times 10^{6}$ c.f.u. were used for both isolates. For the lower number of bacteria (approximately $7 \times 10^{3}$ and $7 \times 10^{4}$ c.f.u.), none of the larvae was dead at 6 days post-infection (data not shown). When approximately $7 \times 10^{5}$ c.f.u. were used virulence attenuation by nonmucoid D2214 was observed, with more than $80 \%$ of the larvae still being alive at 4 days post-infection while in D2095 only $10 \%$ remained alive (Fig. 5b, open symbols). A larger inoculum size (approximately $7 \times 10^{6}$ c.f.u.) also showed virulence attenuation for D2214 when compared with D2095, but in this case larval survival rate was less pronounced in the presence of both isolates (Fig. 5b, filled symbols). These data showing higher survival rates in the presence of D2214 were consistent with the decreased expression of many transcripts encoding proteins putatively involved in virulence that we observed in our microarray data. Nevertheless, with prolonged incubation time and a large inoculum size, both strains display a fully virulent phenotype. 
(a)

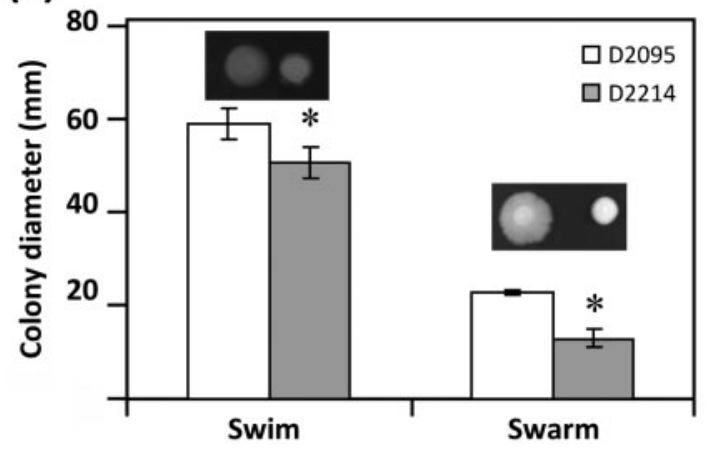

(b)

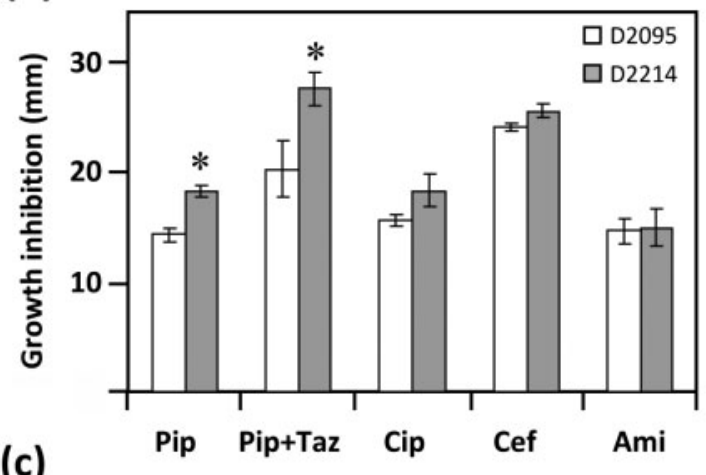

(c)

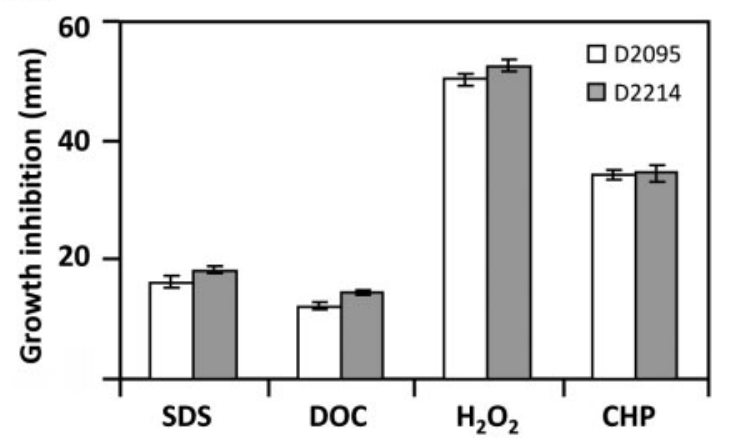

Fig. 4. Phenotypic properties displayed by isolates D2095 and D2214. (a) Swimming and swarming motilities assayed in $0.3 \%$ or $0.5 \%$ agar plates spotted with $5 \mu \mathrm{l}$ bacterial cultures and incubated at $37{ }^{\circ} \mathrm{C}$ for $48 \mathrm{~h}$, followed by measurement of colony diameter. (b) Susceptibility to antibiotics determined at $37{ }^{\circ} \mathrm{C}$ after $24 \mathrm{~h}$ incubation determined by measuring the diameter of cell growth inhibition (Pip, piperacillin; Taz, tazobactam; Cip, ciprofloxacin; Cef, ceftazidime; Ami, amikacin). (c) Sensitivity of isolates to stressinducing agents (10\% SDS, $5 \%$ DOC, $10 \% \mathrm{CHP}$ and $30 \%$ $\mathrm{H}_{2} \mathrm{O}_{2}$ ) determined by measuring growth inhibition haloes after incubation of plates at $37{ }^{\circ} \mathrm{C}$ for $24 \mathrm{~h}$. The data are means $\pm S D$ from the results of at least three independent cell cultivations. The $t$-test was performed using GraphPad Prism 5.0 software. A $P$-value of $<0.013$ was considered significant compared to D2095 $\left(^{*}\right)$.

\section{Genes involved in cell wall biogenesis}

In the category of cell wall biogenesis, the largest group of genes differentially expressed comprises the bce genes (a)

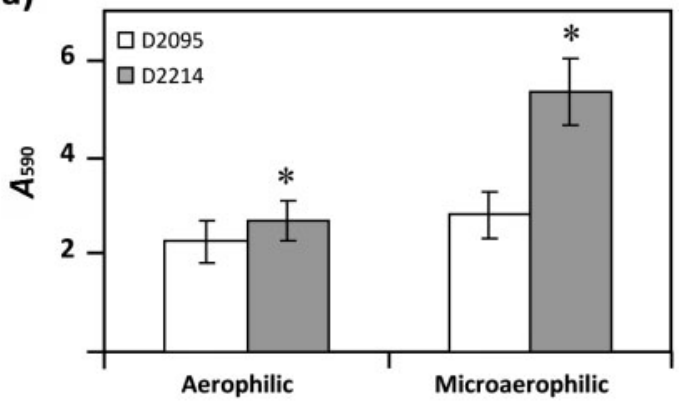

(b)

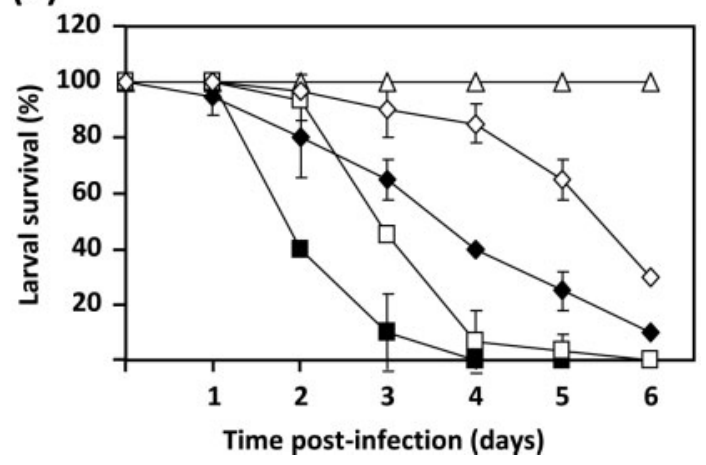

Fig. 5. Comparison between B. multivorans isolates D2095 and D2214 relative to (a) biofilm formation and (b) virulence in Galleria mellonella. The biomass of the biofilm was assessed after $48 \mathrm{~h}$ incubation at $37{ }^{\circ} \mathrm{C}$ in aerophilic and microaerophilic conditions in polystyrene microtitre plates containing SM medium. Virulence tests were performed by injecting $G$. mellonella with $B$. multivorans D2095 using approximately $7 \times 10^{5}(\square)$ and $7 \times 10^{6}(\boldsymbol{\square})$ cells or with $B$. multivorans D2214 using approximately $7 \times 10^{5}(\diamond)$ and $7 \times 10^{6}(\lambda)$ cells, and estimation of larvae survival rates over 6 days. The control experiment without bacteria is also shown $(\triangle)$. The data are means $\pm S D$ from the results of at least three independent experiments. A $P$-value of $<0.01$ was considered significant compared with D2095 $\left(^{*}\right)$.

involved in cepacian biosynthesis (Table 3). All genes from both $b c e-I$ and bce-II gene clusters (Ferreira et al., 2010; Moreira et al., 2003) showed strongly decreased expression in D2214 in comparison to D2095. In fact, 15 out of 19 genes had more than twofold decreased expression. This result partially confirms the non-mucoid phenotype observed for D2214 and suggests that regulation of cepacian biosynthesis in this isolate is probably at the transcriptional level, but post-transcriptional regulation may also account for that phenotype.

Some differences in the outer-membrane composition of the two isolates may also be present since many genes encoding outer-membrane porins showed decreased expression in D2214. These genes are ompW, Bmul_0880, Bmul_2395, Bmul_2847, Bmul_3710 and Bmul_4600. The decrease in the expression levels for porin-encoding genes could indicate a less permeable outer membrane in this isolate. 


\section{Genes involved in transcription and signal transduction}

In the category of transcriptional regulators, the expression of five genes encoding products involved in motility and chemotaxis regulation was found to be decreased in D2214 (Table 3). These genes encode the master regulators FlhCD, the sigma 28 subunit of RNA polymerase FliA, the antisigma $28 \mathrm{FlgM}$ and $\mathrm{CheY}$ protein, which when phosphorylated binds to FliM and changes flagellar motor rotation. As described before, most of the genes under control of these regulators showed decreased expression in D2214.

Genes Bmul_4264 and Bmul_4265, encoding a response regulator receiver protein and a transcriptional regulator of the Crp/Fnr family, respectively, showed decreased expression in the D2214 isolate (Table 3). The Crp (cAMP receptor protein)-like and Fnr (fumarate and nitrate reductase regulatory protein)-like transcription factors comprise proteins which respond directly or indirectly to a broad spectrum of intracellular and exogenous signals (cAMP, oxygen or redox state, oxidative and nitrosative stress, nitric oxide, carbon monoxide, 2-oxoglutarate or temperature) (Körner et al., 2003). Dufour et al. (2010) analysed the Crp/ Fnr conserved regulons within $\alpha$-proteobacteria and found that the core Fnr regulon encodes enzymes for microaerobic or anaerobic respiratory growth, including synthesis of haem, the high-affinity cytochrome $c b b 3$-type oxidase, metal cation transporters, the OmpW porin and the universal stress protein UspA. To our knowledge none of the Burkholderia Crp/Fnr regulators has been characterized, but it is interesting to observe that genes Bmul_2345, encoding a heavy-metal-translocating P-type ATPase, Bmul_5381, encoding a UspA protein, Bmul_0982, encoding a coproporphyrinogen oxidase, ompW and narGHIJ, encoding nitrate reductase, all showed decreased expression in D2214. The decreased expression of genes encoding products such as the Fnr-type regulator and nitrate reductase in D2214 suggests that this isolate is less adapted to survive under low oxygen tension. To test this hypothesis, isolates D2095 and D2214 were grown in SM medium at $37{ }^{\circ} \mathrm{C}$ under microaerophilic conditions. Growth curves (Fig. 1a) confirmed the slower growth rate and lower biomass formed by D2214 when compared with D2095 during the $48 \mathrm{~h}$ of growth.

\section{Genes involved in energy and central intermediary metabolism}

We found decreased expression of several genes involved in central metabolism and energy production in D2214, suggesting lower energetic needs of this isolate. For instance, a decrease of the expression of some smoEFGK genes involved in the uptake of mannitol, the carbon source provided in our experiment, was observed. Similarly, $m t l K$, encoding a mannitol dehydrogenase converting D-mannitol into D-fructose, and the fructokinase-encoding gene Bmul_0700, converting D-fructose into D-fructose 6-phosphate, showed decreased expression in
D2214 (Table 3). Another set of genes encoding a putative monosaccharide transporter encoded by Bmul_3783 to Bmul_3785 (Table 3) showed decreased expression in D2214, reinforcing the idea of reduced sugar uptake needs.

Concerning other carbohydrate metabolism reactions, a decrease in expression of many genes encoding enzymes leading to the cepacian nucleotide sugar precursors was observed. Besides decreased expression of bceACMNT, the expression of genes Bmul_2501 and Bmul_2506, encoding putative UDP-glucose epimerases converting UDP-glucose into UDP-galactose, was decreased in D2214. A set of genes with increased expression in D2214 are putatively involved in osmotic stress response and encode an $\mathrm{ABC}$ transporter (Bmul_3511 and Bmul_3512) and a protein putatively involved in the synthesis of the osmoprotectant $\alpha$-trehalose (otsA) (Table 3). Regarding energy production and conversion, three genes (Bmul_1434-Bmul_1436) encoding products involved in the formation of acetyl-CoA from pyruvate showed decreased expression in D2214 (Table 3). That may indicate a lower availability of acetyl-CoA for the tricarboxylic acid cycle and consequently a reduction of anabolic pathways and ATP synthesis.

Many genes involved in nitrogen metabolism had decreased expression in D2214. That is the case for a cluster of six genes probably involved in anaerobic nitrate respiration. Those genes seem to be an operonic structure composed of narGHJI encoding nitrate reductase, gene Bmul_5387 encoding a peptidyl-prolyl cis-trans isomerase and narK encoding a nitrate/nitrite antiporter. Since gene expression was measured under aerobic conditions, the decreased expression of genes involved in nitrate respiration may suggest that D2214 has a tighter control on the expression of genes whose products are not necessary for bacterial survival, but may also reflect the lower ability to survive under low oxygen as we have shown. Other genes involved in nitrogen metabolism with decreased expression are required for biosynthesis of glutamate, a central metabolite providing nitrogen for the synthesis of all other nitrogen-containing components. These were the glutamate synthase-encoding genes $g l t B D$ as well as glutamate dehydrogenase $g d h A$. Although not to a great extent, the differential expression of genes encoding enzymes for amino acid biosynthesis was primarily decreased in D2214. Decreased expression in D2214 of Bmul_1255, encoding the primary nitrogen sensor $\mathrm{G} \ln \mathrm{D}$, was also observed (Table 3). D2214 probably senses an excess of nitrogen, and therefore many genes related to its metabolism have decreased expression. Together, these results may reflect the lower need for nitrogen and carbon compounds by D2214. This may reflect the lack of EPS biosynthesis burden, although cells were collected several hours before high-molecular-mass EPS was detected in the supernatant, or a broader adaptation mechanism to save energy.

To evaluate whether the metabolic differences observed at the transcriptional level between D2095 and D2214 could give rise to different survival ability, both cultures were 
aerobically incubated for a prolonged period (28 days) under nutrient starvation in minimal medium without a carbon source. C.f.u. counts showed a higher survival rate of D2214 over the full incubation period (Fig. 6).

\section{DISCUSSION}

One of the conclusions derived from our analysis is the confirmation that during the course of chronic lung colonization by Burkholderia, genetic or epigenetic changes leading to development of new bacterial phenotypes arise. These different phenotypes are much more than just mucoid variation and have repercussions in other virulence traits and metabolism. The combined analysis of transcriptional profiling and phenotypic assays allowed us to observe a general reduction of the expression of several virulence factors in the non-mucoid D2214 isolate when compared with the clonal mucoid isolate D2095. This was observed for swimming and swarming motility, type VI secretion, haemolysin-type protein secretion and EPS production. To evaluate whether these changes had an effect on Burkholderia virulence, we chose G. mellonella as an animal model for acute infection assessment (Seed \& Dennis, 2008). Our results showed that D2214 displayed reduced acute virulence when compared with D2095 but it was still able to kill approximately $90 \%$ of the larvae within the time period of the experiment and for the highest cell density tested. Another phenotype displayed by D2214 was a decrease in resistance to some $\beta$-lactam antibiotics. This is an unusual result since due to the antibiotic therapies administered to CF patients bacteria tend to develop higher resistance. The lower resistance level of D2214 isolate against some $\beta$-lactam antibiotics may be due to pleiotropic effects of the mutation(s) that have occurred in this isolate and is most likely not relevant clinically. Isolate

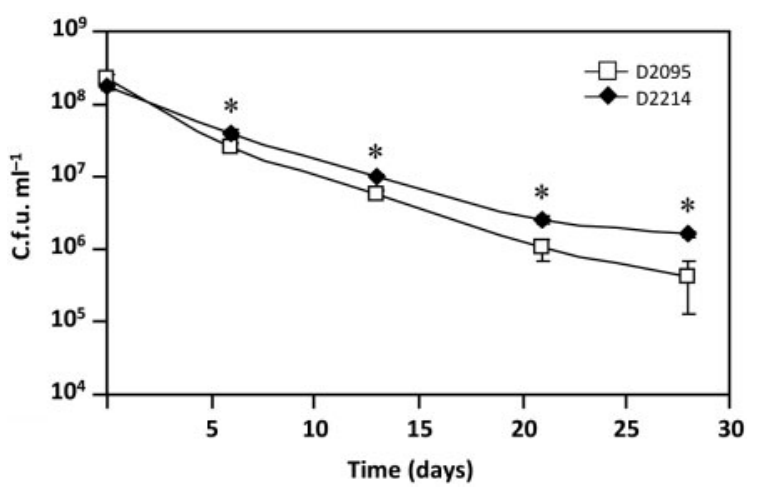

Fig. 6. Ability of B. multivorans isolates D2095 and D2214 to survive starvation in M63 minimal medium without a carbon source. Survival was assessed by c.f.u. determination on LB agar oncubated at $37{ }^{\circ} \mathrm{C}$ for $48 \mathrm{~h}$ under aerobic conditions. Error bars indicate SD. A $P$-value of $<0.034$ was considered significant compared with D2095 (*).
D2214 forms a biofilm with more biomass than D2095 and, despite a lower growth rate under microaerophilic conditions, this effect is more pronounced under this low oxygen tension. This result of biofilm formation ability is in accordance with the lower motility and chemotactic responses displayed by D2214 and may indicate a preference of this bacterium to live a sessile lifestyle, especially if areas of low oxygen tension are available within the lungs and/or chemotactic gradients of nutrients are absent. Similarly, biofilm formation by the non-mucoid $B$. cenocepacia C8963 isolate was shown to be higher than in the highly mucoid C9343, both sequential clonal isolates from a CF patient (Conway et al., 2004). Nevertheless, our result was unexpected due to previous studies showing the positive effect on biofilm formation of the presence of EPS production in B. cepacia IST408 CF isolate (Cunha et al., 2004; Ferreira et al., 2007). It is possible that in D2214, especially under microaerophilic conditions, other determinants of biofilm formation may become relevant.

When growing aerobically in rich medium, D2095 and D2214 showed a similar growth rate. In addition, no significant differences in metabolizing 95 sources of carbon and nitrogen present on Microlog GN2 panels were detected for the two isolates (data not shown). Nevertheless, expression data from cells growing under the same conditions showed decreased expression of many genes involved in carbon and nitrogen metabolism in isolate D2214. This shutdown of unnecessary transcripts and proteins may reflect lower energetic needs since less energy has to be expended on motility and polysaccharide production, two very energetically demanding processes. It is also possible that the decreased expression of many genes involved in metabolism is an adaptation to thrive in an environment, such as the CF lung, where the availability of nutrients varies and other bacterial competitors are present. That could explain the improved survival of D2214 under nutrient starvation for a prolonged period of time.

The first pair of sequential isolates of B. cenocepacia that have been characterized were recovered within 10 months of each other from a chronically infected CF patient (Conway et al., 2004; Zlosnik \& Speert, 2010). The earliest isolate, C8963, was non-mucoid and the latest, C9343, was very mucoid. The comparison of $\mathrm{C} 8963$ with $\mathrm{C} 9343$ by PFGE showed several SpeI-digested chromosomal fragments of a different size (Conway et al., 2004) and C9343 had mutations in the gene cepR, encoding a quorumsensing regulator, and deletion of a region from the pathogenicity island present mostly in $B$. cenocepacia (McKeon et al., 2011; Zlosnik \& Speert, 2010). Our data from the differential genomic DNA hybridization of D2095 and D2214 against the B. multivorans ATCC 17616 genome also demonstrated genetic variation within a region of chromosome 2, resulting in deletion and duplication of genes, confirming that genotypic variation occurs during the course of chronic infection by bacteria. Results from transcriptomic and proteomic analyses in those $B$. cenocepacia isolates showed that the non-mucoid isolate, 
C8963, had increased expression of virulence factors such as the nematocidal protein AidA and the zinc metalloprotease $\mathrm{ZmpA}$, and was more resistant to oxidative stress (Zlosnik \& Speert, 2010). The genes and proteins with lower expression in C8963 were related to metabolism and transport (Zlosnik \& Speert, 2010). In addition, nonmucoid C8963 bound more efficiently to cells of the immune system and displayed a higher clearance rate from the lungs of $\mathrm{BALB} / \mathrm{c}$ mice when compared with the mucoid C9343 isolate (Conway et al., 2004). In our pair of $B$. multivorans isolates D2095 and D2214 we observed the same general decreased expression of genes involved in metabolism and transport functions, but also a clear shutdown of the expression of some genes involved in virulence traits. This probably explains the slower mortality rate that we observed for G. mellonella. Since two different infection models were used to assess virulence of C8963/ C9343 (Conway et al., 2004) and D2095/D2214, it is not possible to compare the results obtained in the two cases.

Considering the mucoid phenotype displayed by isolates $B$. multivorans C5568 to D2095, it is likely that during the 13 years of lung colonization some fitness-improving mutations may have occurred in these bacterial genomes, although they did not impair the bacteria's ability to produce EPS. By contrast, in isolate D2214, additional changes must have occurred so that not only was polysaccharide production ability suppressed but many other phenotypic traits also showed considerable variation. What does this mean in terms of bacterial fitness to persist in the CF lung? Considering that the main characteristics of the CF lung environment are the host immune system, antibiotic therapies and substrate composition, isolate D2214, by its ability to decrease the expression of several virulence traits and perhaps better escape from the immune system, to form more biofilm, and a better management of nutrient resources, may have increased fitness under all those circumstances. These findings are in agreement with the observed inverse correlation between the ability of $B c c$ bacteria to produce EPS and rate of decline in CF lung function (Zlosnik et al., 2011). Nevertheless, other pairs of mucoid/non-mucoid clonal isolates from other Bcc species should be tested to evaluate the possibly increased fitness of the non-mucoid variants.

Data obtained from the transcriptional analysis do not allow us to suggest any specific mechanism behind the morphotype variation observed between D2095 and D2214. It could result from the additive effects of several mutations, or from a mutation in a particular regulatory gene with profound pleiotropic effects. Since hypermutable strains of $P$. aeruginosa are common in persistently infected CF patients (Oliver et al., 2000), we sequenced the mutS and mutL genes, involved in DNA mismatch repair, of D2095 and D2214, but no nucleotide differences were found. Therefore, full DNA sequence determination of both genomes is required. Some other mechanisms responsible for genotypic and phenotypic variation within CF isolates of $P$. aeruginosa obtained from colonized lungs are well known
(Huse et al., 2010; Smith et al., 2006). Genome sequence determination of two $P$. aeruginosa isolates from one CF patient, recovered with a time offset of 90 months, showed several mutations in regulators and virulence factors in the last isolate, including $\mathrm{O}$-antigen biosynthesis, type III secretion, motility, multidrug efflux, osmotic balance, phenazine biosynthesis, quorum sensing and iron acquisition (Smith et al., 2006). Analysis of such mutations in a large collection of isolates showed that the most frequently mutated genes were mexZ, a negative regulator of an efflux pump, and lasR, the primary regulator of quorum sensing (Smith et al., 2006). Another mutation that has stronger implications in disease progression is in the regulatory gene mucA that increases alginate production (Martin et al., 1993; Rau et al., 2010). No protein homologue to anti-sigma factor MucA can be found in Burkholderia genomes and the player(s) directly regulating EPS production in these microorganisms remain(s) unknown. Although the differences at the genetic or epigenetic level giving rise to phenotypic variability in $\mathrm{CF}$ isolates are not yet known, our results point to some mechanisms used by Bcc bacteria for persistence in the lung and will hopefully help to identify vulnerabilities and potential targets for future antimicrobial agent development against these micro-organisms.

\section{ACKNOWLEDGEMENTS}

This work was supported by FEDER and Fundação para a Ciência e a Tecnologia, Portugal (contract PTDC/BIA-MIC/66977/2006, a postdoctoral grant to A. S. F. and doctoral grants to I. N. S. and D. M.-H.).

\section{REFERENCES}

Altschul, S. F., Madden, T. L., Schäffer, A. A., Zhang, J., Zhang, Z., Miller, W. \& Lipman, D. J. (1997). Gapped BLAST and PSI-BLAST: a new generation of protein database search programs. Nucleic Acids Res 25, 3389-3402.

Aubert, D., MacDonald, D. K. \& Valvano, M. A. (2010). BcsKC is an essential protein for the type VI secretion system activity in Burkholderia cenocepacia that forms an outer membrane complex with BcsLB. J Biol Chem 285, 35988-35998.

Baldwin, A., Mahenthiralingam, E., Drevinek, P., Vandamme, P., Govan, J. R., Waine, D. J., LiPuma, J. J., Chiarini, L., Dalmastri, C. \& other authors (2007). Environmental Burkholderia cepacia complex isolates in human infections. Emerg Infect Dis 13, 458-461.

Bauer, A. W., Kirby, W. M., Sherris, J. C. \& Turck, M. (1966). Antibiotic susceptibility testing by a standardized single disk method. Am J Clin Pathol 45, 493-496.

Burtnick, M., Bolton, A., Brett, P., Watanabe, D. \& Woods, D. (2001). Identification of the acid phosphatase (acpA) gene homologues in pathogenic and non-pathogenic Burkholderia spp. facilitates TnphoA mutagenesis. Microbiology 147, 111-120.

Bylund, J., Burgess, L. A., Cescutti, P., Ernst, R. K. \& Speert, D. P. (2006). Exopolysaccharides from Burkholderia cenocepacia inhibit neutrophil chemotaxis and scavenge reactive oxygen species. $J$ Biol Chem 281, 2526-2532.

Coenye, T., Drevinek, P., Mahenthiralingam, E., Shah, S. A., Gill, R. T., Vandamme, P. \& Ussery, D. W. (2007). Identification of putative 
noncoding RNA genes in the Burkholderia cenocepacia J2315 genome. FEMS Microbiol Lett 276, 83-92.

Conway, B. A., Chu, K. K., Bylund, J., Altman, E. \& Speert, D. P. (2004). Production of exopolysaccharide by Burkholderia cenocepacia results in altered cell-surface interactions and altered bacterial clearance in mice. J Infect Dis 190, 957-966.

Costerton, J. W., Stewart, P. S. \& Greenberg, E. P. (1999). Bacterial biofilms: a common cause of persistent infections. Science 284, 13181322.

Cunha, M. V., Sousa, S. A., Leitão, J. H., Moreira, L. M., Videira, P. A. \& Sá-Correia, I. (2004). Studies on the involvement of the exopolysaccharide produced by cystic fibrosis-associated isolates of the Burkholderia cepacia complex in biofilm formation and in persistence of respiratory infections. J Clin Microbiol 42, 3052-3058.

Downey, D. G., Bell, S. C. \& Elborn, J. S. (2009). Neutrophils in cystic fibrosis. Thorax 64, 81-88.

Dufour, Y. S., Kiley, P. J. \& Donohue, T. J. (2010). Reconstruction of the core and extended regulons of global transcription factors. PLoS Genet 6, e1001027.

Ferreira, A. S., Leitão, J. H., Sousa, S. A., Cosme, A. M., Sá-Correia, I. \& Moreira, L. M. (2007). Functional analysis of Burkholderia cepacia genes $b c e D$ and $b c e F$, encoding a phosphotyrosine phosphatase and a tyrosine autokinase, respectively: role in exopolysaccharide biosynthesis and biofilm formation. Appl Environ Microbiol 73, 524-534.

Ferreira, A. S., Leitão, J. H., Silva, I. N., Pinheiro, P. F., Sousa, S. A., Ramos, C. G. \& Moreira, L. M. (2010). Distribution of cepacian biosynthesis genes among environmental and clinical Burkholderia strains and role of cepacian exopolysaccharide in resistance to stress conditions. Appl Environ Microbiol 76, 441-450.

Govan, J. R. \& Nelson, J. W. (1992). Microbiology of lung infection in cystic fibrosis. Br Med Bull 48, 912-930.

Govan, J. R., Brown, P. H., Maddison, J., Doherty, C. J., Nelson, J. W., Dodd, M., Greening, A. P. \& Webb, A. K. (1993). Evidence for transmission of Pseudomonas cepacia by social contact in cystic fibrosis. Lancet 342, 15-19.

Hammond, J. P., Broadley, M. R., Craigon, D. J., Higgins, J., Emmerson, Z. F., Townsend, H. J., White, P. J. \& May, S. T. (2005). Using genomic DNA-based probe-selection to improve the sensitivity of high-density oligonucleotide arrays when applied to heterologous species. Plant Methods 1, 10.

He, J., Dai, X. \& Zhao, X. (2007). PLAN: a web platform for automating high-throughput BLAST searches and for managing and mining results. BMC Bioinformatics 8, 53.

Holden, M. T., Seth-Smith, H. M., Crossman, L. C., Sebaihia, M., Bentley, S. D., Cerdeño-Tárraga, A. M., Thomson, N. R., Bason, N., Quail, M. A. \& other authors (2009). The genome of Burkholderia cenocepacia J2315, an epidemic pathogen of cystic fibrosis patients. J Bacteriol 191, 261-277.

Hunt, T. A., Kooi, C., Sokol, P. A. \& Valvano, M. A. (2004). Identification of Burkholderia cenocepacia genes required for bacterial survival in vivo. Infect Immun 72, 4010-4022.

Huse, H. K., Kwon, T., Zlosnik, J. E., Speert, D. P., Marcotte, E. M. \& Whiteley, M. (2010). Parallel evolution in Pseudomonas aeruginosa over 39,000 generations in vivo. MBio 1, e00199-e00210.

Isles, A., Maclusky, I., Corey, M., Gold, R., Prober, C., Fleming, P. \& Levison, H. (1984). Pseudomonas cepacia infection in cystic fibrosis: an emerging problem. J Pediatr 104, 206-210.

Körner, H., Sofia, H. J. \& Zumft, W. G. (2003). Phylogeny of the bacterial superfamily of Crp-Fnr transcription regulators: exploiting the metabolic spectrum by controlling alternative gene programs. FEMS Microbiol Rev 27, 559-592.
Leitão, J. H., Sousa, S. A., Ferreira, A. S., Ramos, C. G., Silva, I. N. \& Moreira, L. M. (2010). Pathogenicity, virulence factors, and strategies to fight against Burkholderia cepacia complex pathogens and related species. Appl Microbiol Biotechnol 87, 31-40.

Li, C. \& Wong, W. H. (2001a). Model-based analysis of oligonucleotide arrays: model validation, design issues and standard error application. Genome Biol 2, H0032.

Li, C. \& Wong, W. H. (2001b). Model-based analysis of oligonucleotide arrays: expression index computation and outlier detection. Proc Natl Acad Sci U S A 98, 31-36.

Mahenthiralingam, E., Urban, T. A. \& Goldberg, J. B. (2005). The multifarious, multireplicon Burkholderia cepacia complex. Nat Rev Microbiol 3, 144-156.

Martin, D. W., Schurr, M. J., Mudd, M. H., Govan, J. R., Holloway, B. W. \& Deretic, V. (1993). Mechanism of conversion to mucoidy in Pseudomonas aeruginosa infecting cystic fibrosis patients. Proc Natl Acad Sci U S A 90, 8377-8381.

McKeon, S. A., Nguyen, D. T., Viteri, D. F., Zlosnik, J. E. \& Sokol, P. A. (2011). Functional quorum sensing systems are maintained during chronic Burkholderia cepacia complex infections in patients with cystic fibrosis. J Infect Dis 203, 383-392.

Moreira, L. M., Da Costa, M. S. \& Sá-Correia, I. (1997). Comparative genomic analysis of isolates belonging to the six species of the genus Thermus using pulsed-field gel electrophoresis and ribotyping. Arch Microbiol 168, 92-101.

Moreira, L. M., Videira, P. A., Sousa, S. A., Leitão, J. H., Cunha, M. V. \& Sá-Correia, I. (2003). Identification and physical organization of the gene cluster involved in the biosynthesis of Burkholderia cepacia complex exopolysaccharide. Biochem Biophys Res Commun 312, 323333.

Nzula, S., Vandamme, P. \& Govan, J. R. (2002). Influence of taxonomic status on the in vitro antimicrobial susceptibility of the Burkholderia cepacia complex. J Antimicrob Chemother 50, 265269.

Oliver, A., Cantón, R., Campo, P., Baquero, F. \& Blázquez, J. (2000). High frequency of hypermutable Pseudomonas aeruginosa in cystic fibrosis lung infection. Science 288, 1251-1253.

Pedersen, S. S., Høiby, N., Espersen, F. \& Koch, C. (1992). Role of alginate in infection with mucoid Pseudomonas aeruginosa in cystic fibrosis. Thorax 47, 6-13.

Pfaffl, M. W. (2001). A new mathematical model for relative quantification in real-time RT-PCR. Nucleic Acids Res 29, e45.

Rau, M. H., Hansen, S. K., Johansen, H. K., Thomsen, L. E., Workman, C. T., Nielsen, K. F., Jelsbak, L., Høiby, N., Yang, L. \& Molin, S. (2010). Early adaptive developments of Pseudomonas aeruginosa after the transition from life in the environment to persistent colonization in the airways of human cystic fibrosis hosts. Environ Microbiol 12, 1643-1658.

Sambrook, J. \& Russell, D. W. (2001). Molecular Cloning: a Laboratory Manual. Cold Spring Harbor, NY: CSHL Press.

Schwarz, S., Hood, R. D. \& Mougous, J. D. (2010). What is type VI secretion doing in all those bugs? Trends Microbiol 18, 531537.

Seed, K. D. \& Dennis, J. J. (2008). Development of Galleria mellonella as an alternative infection model for the Burkholderia cepacia complex. Infect Immun 76, 1267-1275.

Smith, E. E., Buckley, D. G., Wu, Z., Saenphimmachak, C., Hoffman, L. R., D’Argenio, D. A., Miller, S. I., Ramsey, B. W., Speert, D. P. \& other authors (2006). Genetic adaptation by Pseudomonas aeruginosa to the airways of cystic fibrosis patients. Proc Natl Acad Sci U S A 103, 8487-8492. 
Sousa, S. A., Ulrich, M., Bragonzi, A., Burke, M., Worlitzsch, D., Leitão, J. H., Meisner, C., Eberl, L., Sá-Correia, I. \& Döring, G. (2007). Virulence of Burkholderia cepacia complex strains in gp91 ${ }^{\text {phox-l- }}$ mice. Cell Microbiol 9, 2817-2825.

Taylor, B. L. (2007). Aer on the inside looking out: paradigm for a PAS-HAMP role in sensing oxygen, redox and energy. Mol Microbiol 65, 1415-1424.

Zlosnik, J. E. \& Speert, D. P. (2010). The role of mucoidy in virulence of bacteria from the Burkholderia cepacia complex: a systematic proteomic and transcriptomic analysis. J Infect Dis 202, 770-781.
Zlosnik, J. E., Hird, T. J., Fraenkel, M. C., Moreira, L. M., Henry, D. A. \& Speert, D. P. (2008). Differential mucoid exopolysaccharide production by members of the Burkholderia cepacia complex. J Clin Microbiol 46, 1470-1473.

Zlosnik, J. E., Costa, P. S., Brant, R., Mori, P. Y., Hird, T. J., Fraenkel, M. C., Wilcox, P. G., Davidson, A. G. \& Speert, D. P. (2011). Mucoid and nonmucoid Burkholderia cepacia complex bacteria in cystic fibrosis infections. Am J Respir Crit Care Med 183, 67-72.

Edited by: M. Whiteley 